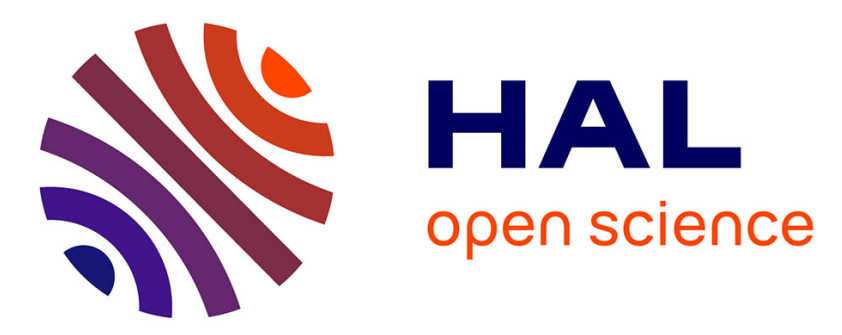

\title{
A Multiscale Modeling of Magnetic Shape Memory Alloys: Application to NiMnGa Single Crystal
}

Mame Daro Fall, Olivier Hubert, Frédéric Mazaleyrat, K Lavernhe-Taillard, A Pasko

\section{To cite this version:}

Mame Daro Fall, Olivier Hubert, Frédéric Mazaleyrat, K Lavernhe-Taillard, A Pasko. A Multiscale Modeling of Magnetic Shape Memory Alloys: Application to NiMnGa Single Crystal. IEEE Transactions on Magnetics, 2016, 52, pp.1 - 4. 10.1109/TMAG.2016.2514705 . hal-01532881

\section{HAL Id: hal-01532881 https://hal.science/hal-01532881}

Submitted on 3 Jun 2017

HAL is a multi-disciplinary open access archive for the deposit and dissemination of scientific research documents, whether they are published or not. The documents may come from teaching and research institutions in France or abroad, or from public or private research centers.
L'archive ouverte pluridisciplinaire HAL, est destinée au dépôt et à la diffusion de documents scientifiques de niveau recherche, publiés ou non, émanant des établissements d'enseignement et de recherche français ou étrangers, des laboratoires publics ou privés. 


\title{
A multiscale modeling of magnetic shape memory alloys: Application to NiMnGa single crystal
}

\author{
M. D. Fall*, O. Hubert*, F. Mazaleyrat ${ }^{\dagger}$, K. Lavernhe-Taillard*, and A. Pasko ${ }^{\dagger}$ \\ *LMT-Cachan, ENS Cachan / CNRS UMR 8535 / Université Paris-Saclay \\ 61 Avenue du Président Wilson, F-94235 Cachan Cedex, France \\ †SATIE, ENS Cachan / CNRS UMR 8029 / Université Paris-Saclay \\ 61 Avenue du Président Wilson, F-94235 Cachan Cedex, France
}

\begin{abstract}
The paper presents a new multiscale modeling dedicated to magnetic shape memory alloys. It involves four scales from the domain scale to the macroscale. The model is presented and simulation results are compared to experiments carried out on a NiMnGa single crystal.

Index Terms-Multiscale Modeling, Magnetic Shape Memory Alloys (MSMA), Chemo-mechanical Coupling, Magnetomechanical Coupling.
\end{abstract}

\section{INTRODUCTION}

Magnetic shape memory alloys (MSMAs) are among the most promising alloys for designing miniature sensors or actuators [1] especially because they are home to a strong thermomagneto-mechanical coupling involving a phase change and/or variant selection. Besides stress and temperature dependence, a macroscopic deformation can be achieved by applying a magnetic field. The magnetization process is the result of three competing mechanisms [2]: the motion of $180^{\circ}$ magnetic domain walls, the local rotation of magnetization vectors and magnetic field induced variants' reorientation. This point is illustrated in figure 1 in the case of a cubic austenite and three possible tetragonal martensite variants [3]. The two first mechanisms exist in common ferromagnetic materials. The first mechanism do not lead to any deformation, the second do not lead to large deformation since only the spin-orbit coupling is involved leading to the so-called magnetostriction. Only the last one that mingles with a $90^{\circ}$ magnetic domain walls motion (existing in common ferromagnetic material and leading to magnetostriction) leads to large deformation that may reach up to $12 \%$ in single crystals [4], and by consequence to a strong magnetoelastic coupling. The main interest of this mechanism is that it corresponds to a second order thermodynamic transformation that do not involves heat exchange. This is the reason why MSMAs may be used at higher frequencies than common shape memory alloys (up to $1 \mathrm{kHz}$ ) [2]. The piezomagnetic effect in these materials can also be very high allowing the conception of energy harvesting systems [5]. Since these behaviors are strongly associated to transformations at the single crystal scale, development of micro-macro approaches is relevant for their modeling. In this paper, we give first the principles of a unified multiscale modeling of these materials using a homogenization method and a stochastic approach as a basis of the constitutive law
[6] [7]. The model is based on the comparison of the free energy of each domain at the variant scale and each variant at the single crystal scale, and the calculation of their associated volume fractions. Averaging operations allow the calculation of the macroscopic quantities at the polycrystalline scale. The modeling is applied to the prevision of NiMnGa single crystal MSMA behavior. Numerical results are compared to experimental results illustrating the wide variety of phenomena associated with magneto-mechanical and chemo-mechanical couplings.

a

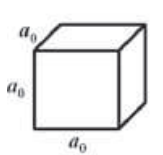

Cubic austenite

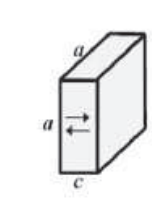

b
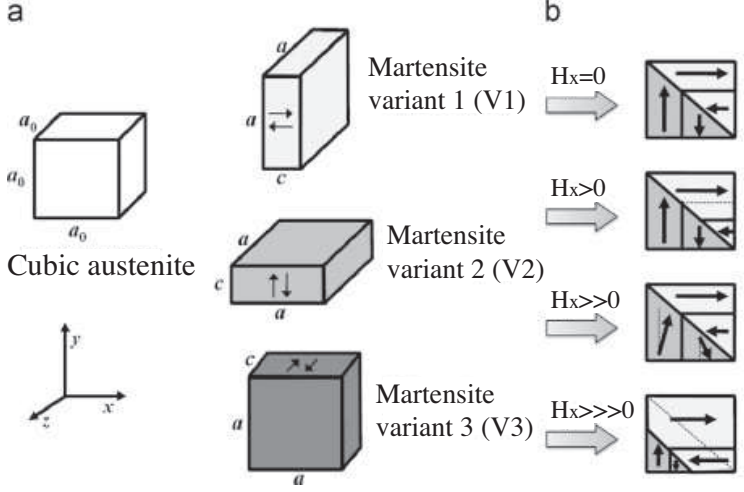

$\mathrm{V} 1+\mathrm{V} 2$

Magnetic

domain

wall motion

Magnetization rotation

Fig. 1. (a) Cubic austenite (cell parameter $a_{0}$ ) and the 3 tetragonal martensite variants (cell parameters $c$ and $a$ ) (b) Magnetization process of (V1+V2) single crystal [3].

\section{Multiscale modeling}

The proposed multiscale model is relevant for multiaxial stress, thermal and magnetic fields loadings in a pseudoreversible framework (heat exchange can be considered for instance). It derives from the previous works of Daniel and Maynadier [6], [7]. The first scale involved for which magnetic, mechanical and thermal quantities can be considered homogeneous is the domain scale inside the variant; just above, the variant scale (including austenite phase) is the scale where mechanical and thermal quantities can be considered homogeneous. The third scale is the grain scale considered as an assembly of variants. The last scale is the polycrystalline scale considered as an assembly of grains and usually denoting the representative volume element (RVE). 


\section{A. Variants free energy}

In the following, $\phi$ denotes a variant, meaning a martensite variant or the austenite phase; $\phi^{\alpha}$ denotes a domain family $\alpha$ inside the variant $\phi$ (e.g. variants and domains illustrated in figure 1). According to the chemo-magneto-mechanical coupling and neglecting the boundary effects, the free energy of a domain family is given by (1). $W_{\phi^{\alpha}}^{T}$ indicates the chemical energy (2), considered to exhibit a linear dependance with temperature. $W_{\phi^{\alpha}}^{\sigma}$ indicates the elastic energy (3). $W_{\phi^{\alpha}}^{\text {mag }}$ indicates the magnetic energy (4).

$$
\begin{gathered}
W_{\phi^{\alpha}}=W_{\phi^{\alpha}}^{T}+W_{\phi^{\alpha}}^{\sigma}+W_{\phi^{\alpha}}^{m a g} \\
W_{\phi^{\alpha}}^{T}=h_{\phi}-T_{\phi^{\alpha}} s_{\phi} \\
W_{\phi^{\alpha}}^{\sigma}=\frac{1}{2} \sigma_{\phi^{\alpha}}: \mathbb{C}_{\phi}^{-1}: \boldsymbol{\sigma}_{\phi^{\alpha}} \\
W_{\phi^{\alpha}}^{m a g}=W_{\phi^{\alpha}}^{H}+W_{\phi^{\alpha}}^{K}
\end{gathered}
$$

$h_{\phi}$ and $s_{\phi}$ are the volumetric enthalpy and entropy $\left(\mathrm{J} . \mathrm{m}^{-3}\right)$ : they do not differ from one domain to another or from a martensite variant to another but differ from martensite to austenite. $T_{\phi^{\alpha}}$ is the local temperature $(\mathrm{K}) . \sigma_{\phi^{\alpha}}$ and $\mathbb{C}_{\phi}$ are the local stress and the stiffness tensor. $\mathbb{C}_{\phi}$ does not differ from one domain to another but strictly differs from one variant to another (due to change of crystal orientation) and from martensite to austenite.

The magnetic energy $W_{\phi^{\alpha}}^{m a g}$ (4) is a combination of the magnetostatic Zeeman energy $\left(W_{\phi^{\alpha}}^{H}\right)$ and magneto crystalline anisotropy energy $\left(W_{\phi^{\alpha}}^{K}\right)$. Exchange energy is not considered because of the uniformity of magnetization over a domain (domain wall contribution is neglected). Zeeman (magnetostatic) energy is directly associated to local magnetic field $\vec{H}_{\phi^{\alpha}}$ (5) (with $\mu_{0}$ the vacuum permeability). This energy tends to align the magnetization at the domain scale $\left(\vec{M}_{\phi^{\alpha}}\right)$ with the applied magnetic field. Magneto crystalline anisotropy energy $\left(W_{\phi^{\alpha}}^{K}\right)$ tends to align magnetization along the crystal easy axes. The easy axes of magnetization are associated to the crystal symmetry. Equation (6) gives $W_{\phi^{\alpha}}^{K}$ for a cubic symmetry (corresponding to austenite in the present case) and equation (7) gives $W_{\phi^{\alpha}}^{K}$ for a uniaxial symmetry of axis $\vec{e}_{u}$ (corresponding to martensite in the present case).

$$
\begin{gathered}
W_{\phi^{\alpha}}^{H}=-\mu_{0} \vec{H}_{\phi^{\alpha}} \cdot \vec{M}_{\phi^{\alpha}} \\
W_{\phi^{\alpha}}^{K}=K_{1}\left(\gamma_{1}^{2} \gamma_{2}^{2}+\gamma_{2}^{2} \gamma_{3}^{2}+\gamma_{3}^{2} \gamma_{1}^{2}\right)+K_{2}\left(\gamma_{1}^{2} \gamma_{2}^{2} \gamma_{3}^{2}\right) \\
W_{\phi^{\alpha}}^{K}=K_{0}\left(1-\gamma_{u}^{2}\right)
\end{gathered}
$$

$K_{0}, K_{1}$ and $K_{2}$ are the magneto-crystalline anisotropy constants. $\gamma_{i}$ are the direction cosines of the local magnetization so that $\vec{M}_{\phi^{\alpha}}=M_{s \phi} \cdot \gamma_{i} \vec{e}_{i}$. The saturation magnetization $M_{s \phi}$ does not differ from one domain to another or from a martensite variant to another but differ from martensite to austenite.

\section{B. Constitutive law}

The internal variables of the problem are the volume fraction of domain families $f_{\phi^{\alpha}}$ and the orientation of the magnetization inside each domain family (two spherical angles per domain family: $\theta_{\phi^{\alpha}}$ and $\psi_{\phi^{\alpha}}$ ). The total number of internal variables strongly depends on the number of variants $(n)$ and number of domains inside each variant $(m)$. Following [6], we use a probabilistic Boltzmann function to calculate the volume fraction and implement an energy minimization for the determination of magnetization direction. The volume fraction $f_{\phi^{\alpha}}$ is given by (8) where $A_{s}$ is an adjusting parameter.

$$
f_{\phi^{\alpha}}=\frac{\exp \left(-A_{s} W_{\phi^{\alpha}}\right)}{\sum_{\phi=1}^{n} \sum_{\alpha=1}^{m} \exp \left(-A_{s} W_{\phi^{\alpha}}\right)}
$$

This general formulation does not allow to make the difference between a domain wall motion inside a variant or a phase, and a domain wall motion associated to a variant reorientation. Indeed, interfacial energies are strongly different [2]. We have to separate the phenomena dominant at the variant scale from the phenomena dominant at the domain scale. The new volume fraction $f_{\phi^{\alpha}}$ given by (9) is using two different parameters $A$ and $B$ driving the two different interfacial effects (indeed $A$ and $B$ parameters introduce the inertial effects ignored by the modeling [6]).

$$
f_{\phi^{\alpha}}=\frac{\sum_{\alpha=1}^{m} \exp \left(-A W_{\phi^{\alpha}}\right)}{\sum_{\phi=1}^{n} \sum_{\alpha=1}^{m} \exp \left(-A W_{\phi^{\alpha}}\right)} \cdot \frac{\exp \left(-B W_{\phi^{\alpha}}\right)}{\sum_{\alpha=1}^{m} \exp \left(-B W_{\phi^{\alpha}}\right)}
$$

It can be noticed that for $A=B$, equation (8) is recovered. Moreover the volume fraction of a variant $\phi$ is simply given by:

$$
f_{\phi}=\sum_{\alpha=1}^{m} f_{\phi^{\alpha}}=\frac{\sum_{\alpha=1}^{m} \exp \left(-A W_{\phi^{\alpha}}\right)}{\sum_{\phi=1}^{n} \sum_{\alpha=1}^{m} \exp \left(-A W_{\phi^{\alpha}}\right)}
$$

Equation (9) is complemented by the minimization operation reported in equation (11):

$$
\left\{\theta_{\phi^{\alpha}}, \psi_{\phi^{\alpha}}\right\}=\min \left(W_{\phi^{\alpha}}\right)
$$

\section{Scale transition rules}

Macroscopic loading are temperature $T$, stress $\sigma$ and magnetic field $\vec{H}$. The macroscopic responses we want to calculate are the transformation deformation $\varepsilon^{t r}$ and the magnetization $\vec{M}$. Some localization rules are required to write the loadings at the appropriate scale and homogenization rules to make the averaging operations.

Temperature is taken uniform over the domain, variant, grain and $\operatorname{RVE}\left(T_{\phi^{\alpha}}=T_{\phi}=T_{g}=T\right)$. This hypothesis can apply even if heat exchanges are considered because MSMAs are metallic materials exhibiting high thermal conductivity and that RVE size is considered as a volume smaller than $1 \mathrm{~mm}^{3}$. Determination of the stress at the domain scale requires a localization process thanks to Hill's formulation. The domain is considered as an inclusion inside the grain defining the equivalent medium. The total deformation at the domain scale $\varepsilon_{\phi^{\alpha}}$ is given by (12) as a sum of elastic $\varepsilon_{\phi^{\alpha}}^{e}$, phase 
transformation $\varepsilon_{\phi^{\alpha}}^{t}$ and magnetostriction $\varepsilon_{\phi^{\alpha}}^{\mu}$ deformations. The latter is very small with respect to transformation strain and is thus neglected for martensite variants. It is taken in account for austenite phase whose transformation strain is null.

$$
\varepsilon_{\phi^{\alpha}}=\varepsilon_{\phi^{\alpha}}^{e}+\varepsilon_{\phi^{\alpha}}^{t}+\varepsilon_{\phi^{\alpha}}^{\mu}
$$

We define the total transformation deformation at the domain scale by: $\varepsilon_{\phi^{\alpha}}^{t r}=\varepsilon_{\phi^{\alpha}}^{t}+\varepsilon_{\phi^{\alpha}}^{\mu}$. It is defined at the grain scale by $\varepsilon_{g}^{t r}=\sum_{\phi=1}^{n} \sum_{\alpha=1}^{m} f_{\phi^{\alpha}} \varepsilon_{\phi^{\alpha}}^{t r}$ (experimental data required to calculate these tensors can easily be found in literature). An homogeneous deformation hypothesis is employed [6] giving the local stress (13), that strongly simplifies the elastic energy expression [7].

$$
\boldsymbol{\sigma}_{\phi^{\alpha}}=\boldsymbol{\sigma}_{g}+\mathbb{C}_{\phi}:\left(\varepsilon_{g}^{t r}-\varepsilon_{\phi^{\alpha}}^{t r}\right)
$$

$\boldsymbol{\sigma}_{g}$ is the stress at the single crystal scale. It is defined as function of macroscopic stress $\sigma$, macroscopic transformation deformation $\varepsilon^{t r}$, stress concentration tensor $\mathbb{B}$ and accommodation stiffness tensor $\mathbb{C}_{a c c}$ (see [6] for detailed expressions) following:

$$
\boldsymbol{\sigma}_{g}=\mathbb{B}: \boldsymbol{\sigma}+\mathbb{C}_{a c c}:\left(\varepsilon^{t r}-\varepsilon_{g}^{t r}\right)
$$

The strong fluctuations of variant and domain volume fraction inside a grain do not allow us to define some admissible localization rules for the magnetic field. It is consequently considered uniform over the grain: $\vec{H}_{\phi^{\alpha}}=\vec{H}_{\phi}=\vec{H}_{g}$. Following [6], a grain can be considered as a sphere inside the RVE. The magnetic field at the single crystal scale $\vec{H}_{g}$ can consequently be deduced from the macroscopic magnetic field, the magnetization at the grain scale $\vec{M}_{g}$ (that verifies: $\vec{M}_{g}=\sum_{\phi=1}^{n} \sum_{\alpha=1}^{m} f_{\phi^{\alpha}} \vec{M}_{\phi^{\alpha}}$, the macroscopic magnetization and the secant macroscopic susceptibility $\chi_{m}$ (with $\chi_{m}=$ $\|\vec{M}\| /\|\vec{H}\|)$ :

$$
\vec{H}_{g}=\vec{H}+\frac{1}{3+2 \chi_{m}}\left(\vec{M}-\vec{M}_{g}\right)
$$

It must be noticed that localization rules (14) and (15) suppose a clear scale separation between grain and RVE sizes (see [6] for more details concerning that point). Averaging operations consist in the calculation of the transformation strain and magnetization at the grain scale (already given above), and the transformation strain and magnetization at the RVE scale. The latter are given in (16) and (17) with $N$ denoting the number of grains. The RVE is practically an orientation data file representative of the material that may be obtained thanks to electron backscattered diffraction (EBSD).

$$
\begin{gathered}
\varepsilon^{t r}=<{ }^{t} \mathbb{B}: \varepsilon_{g}^{t r}>=\frac{1}{N} \sum_{N}{ }^{t} \mathbb{B}: \varepsilon_{g}^{t r} \\
\vec{M}=<\vec{M}_{g}>=\frac{1}{N} \sum_{N} \vec{M}_{g}
\end{gathered}
$$

The multiscale model is a self-consistent modeling. It has been implemented in MATLAB. The time calculation of one loading point for a RVE of about 100 grains is less than 1 minute using a personal computer.

\section{EXPERIMENTS}

Experiments have been carried out using a $\mathrm{Ni}_{49.7 \%} \mathrm{Mn}_{29.5 \%} \mathrm{Ga}_{20.8 \%}$ Heusler single crystal (percentages are given in at\%). Information about thermal transitions and associated enthalpies have been obtained thanks to a Differential Scanning Calorimetry (DSC) analysis. This measurement shows that the material is fully martensitic at room temperature. Moreover the hysteresis associated to the phase transition $\left(\mathrm{T}_{\text {aust } \rightarrow \text { mart }}=45^{\circ} \mathrm{C}\right.$ and $\mathrm{T}_{\text {mart } \rightarrow \text { aust }}=54.9^{\circ} \mathrm{C}$ ) and the Curie temperature $\left(\approx 103^{\circ} \mathrm{C}\right)$ appears clearly. The DSC measurement allows to identify the enthalpies and entropies of austenite and martensite phases (using the temperature transitions and enthalpy variation $\Delta H$ estimated at $34.4 \times 10^{6} \mathrm{~J} \mathrm{~m}^{-3}$ ). Values are consistent with those reported in [8] for 5M martensite. The martensite structure appears to be tetragonal according to XRD and EBSD measurements. The single crystal is on the other hand made of a single variant (2 magnetic domains) whose short axis $\vec{c}$ is aligned with the specimen longitudinal axis $\vec{x}$ (i.e. variant V1 in figure 1a).

Magnetization tests have been carried out using a cubic specimen $\left(2 \times 2 \times 2 \mathrm{~mm}^{3}\right)$. They have been made at room temperature using a Vibrating Sample Magnetization (VSM). Fig 2a shows the initial magnetization curve obtained along the easy axis. This curve is quite different from those seen in the literature [9] for 5M martensite. Indeed EBSD measurements showed that the cube exhibits a low percentage of the two other variants (V2 and V3), whose appearance may be due to the cutting process. After reinitialization (heat treatment and magnetic saturation along $\vec{x}$ axis), a second set of measurements has been carried out. They are reported in figure $2 b$. The material exhibits now two hard axis of magnetization (along the long axis of the tetragonal cell - directions $\vec{y}$ and $\vec{z}$ - only $\vec{y}$ measurement is reported) and one clear easy axis of magnetization (along the short axis of the tetragonal cell - direction $\vec{x}$ ). $180^{\circ}$ domain wall motion is dominant for magnetization along $\vec{x}$ direction; martensite reorientation concomitant to magnetization rotation occurs for magnetization along $\vec{y}$ direction. These new results are in accordance with literature. magnetic measurement carried at room temperature and at $70^{\circ} \mathrm{C}$ allowed on the other hand to identify the magnetic saturation of martensite $\left(\mathrm{M}_{s}^{M}=5.5 \times 10^{5} \mathrm{~A} / \mathrm{m}\right)$ and austenite $\left(\mathrm{M}_{s}^{A}=4.0 \times 10^{5} \mathrm{~A} / \mathrm{m}\right)$.

\section{Modeling}

The modeling concerns the NiMnGa single crystal. The RVE scale is consequently the same than the single crystal scale. The multiscale modeling presented above requires many parameters: most of these parameters are physical parameters that can be found in literature or identified using the few experimental results we reported. Table I gathers the parameters used. The only adjusting parameters are $A$ and $B$. $A$ has been identified using the DSC measurement (adjustment of peak size and shape): figure 3 shows a zoom of the DSC experimental curve (heating) compared to the modeling, given by equation (18) derived from heat equation in adiabatic 

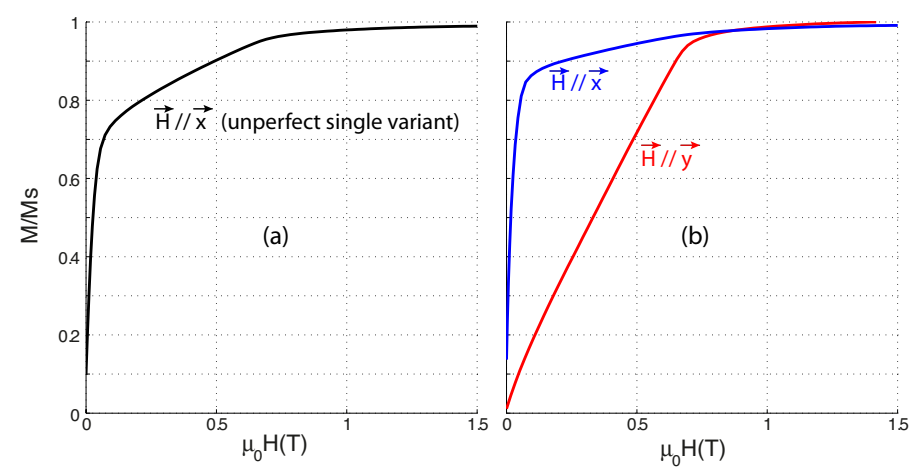

Fig. 2. Magnetization curves obtained by VSM: (a) initial easy axis $\vec{x}$ measurement: multivariants single crystal; (b) easy axis $\vec{x}$ and hard axis $\vec{y}$ measurement after reinitialization of the material: single variant single crystal.

condition (with: $Q$ the heat flow; $d f_{M}$ the martensite fraction increment, obtained from the multiscale modeling, associated to the temperature increment $d T ; \dot{T}$ the temperature rate and $V$ the volume of material used during DSC experiments).

$$
Q=-\frac{d f_{M}}{d T} \dot{T} \Delta H V
$$

$B$ parameter is identified using a single magnetization curve ( $B$ is proportional to the initial susceptibility [6]). Another important point is the choice of a configuration stress (as introduced in [10]), leading to a first set of selected variant. Indeed, without configuration (residual stress), the variants are equiprobable in contradiction with experiments. Figure 4a shows the modeling of magnetization curves obtained at room temperature. A compression stress of $\sigma=-3 \mathrm{MPa}$ along $\vec{x}$ axis allows a strong selection of variant V1: magnetization curves along $\vec{x}$ and $\vec{y}$ are in good agreement with experiments (figure 2a). A compression stress of -1MPa along $\vec{x}$ leads to a lower selection (V2 and V3 are still present). The simulation corresponds now to the initial experimental magnetization curve along $\vec{x}$ (figure 2a). The associated deformation curves are plotted in figure $4 \mathrm{~b}$. They are in accordance with results from literature [9].

\begin{tabular}{|c||c|c|}
\hline Param. & Aust. & Mart. \\
\hline Lat. param. $(\mathrm{nm})-\mathrm{XRD}$ & $\mathrm{a}_{0}=0.582$ & $\mathrm{a}=0.594, \mathrm{c}=0.554$ \\
$\mathrm{M}_{s}\left(\times 10^{5} \mathrm{~A} / \mathrm{m}\right)-\mathrm{VSM}$ & 4 & 5.5 \\
$K_{i}\left(\mathrm{~kJ} . \mathrm{m}^{-3}\right)$ & $\left(\mathrm{K}_{1}=0.3, \mathrm{~K}_{2}=0\right)[11]$ & $\mathrm{K}_{0}=170[12]$ \\
$h_{\phi}\left(\times 10^{6} \mathrm{~J}^{-3}\right)-\mathrm{DSC}$ & -110 & -144.4 \\
$s_{\phi}\left(\mathrm{kJ}^{-3} \mathrm{~m}^{-3}\right)-\mathrm{DSC}$ & 328 & 200 \\
\hline Stiffness $\left(\mathrm{C}_{11}, \mathrm{C}_{12}, C_{44}\right)(\mathrm{GPa})$ & \multicolumn{2}{|c|}{$(144,132,104)[11]$} \\
$(A, B)\left(\times 10^{-4} \mathrm{~m}^{3} / \mathrm{J}\right)$ & $(0.3,1.5)$ \\
\hline
\end{tabular}

TABLE I

PARAMETERS OF THE MULTISCALE MODEL

\section{CONClusion}

A multiscale modeling of MSMA has been proposed. It is a fully thermo-magneto-mechanical coupled model that allows to simulate the (pseudo) reversible behavior of various MSMA single- or poly- crystals (phase constitution, mechanical and

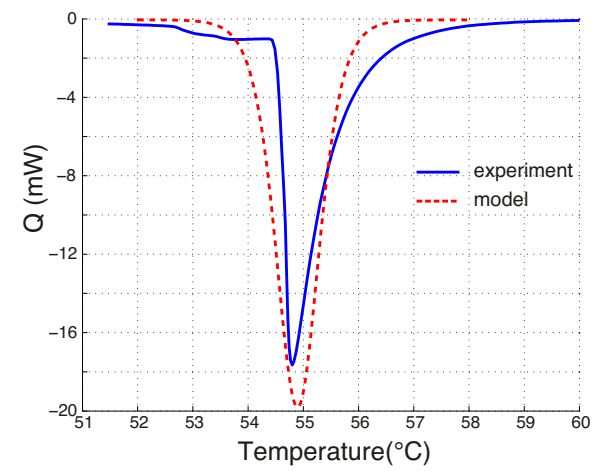

Fig. 3. Comparison model/ experiment of martensite to austenite transition DSC peak.
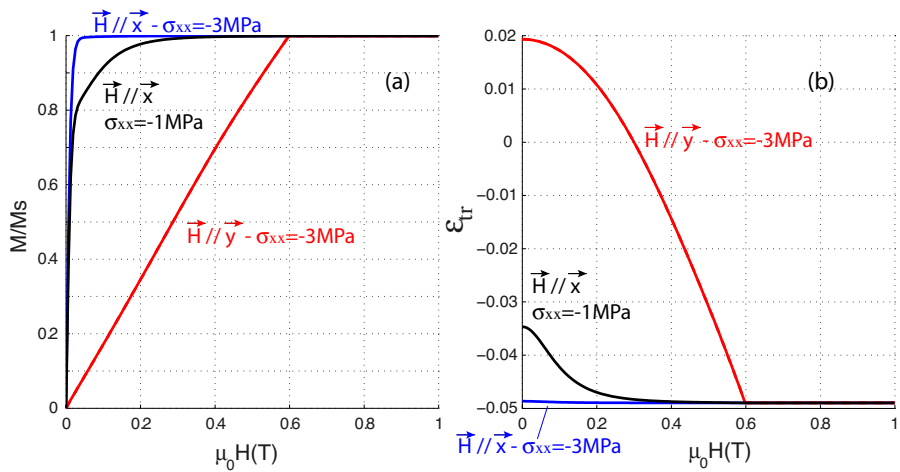

Fig. 4. Modeling results: magnetization curves with magnetic field applied along $\vec{x}$ and $\vec{y}$ axis: effect of configuration stress: $-3 \mathrm{MPa}$ and $-1 \mathrm{MPa}$.

magnetic behaviors) under various loading conditions (including multiaxial stress). Extension to irreversible phenomena and quantitative validations through deformation measurements under magnetic field and biaxial stress are planned. They will be complemented by in situ X-Ray measurements.

\section{REFERENCES}

[1] D.C. Jiles, C.C.H. Lo, Sensors and Actuators, A 106 (2003), 3-7.

[2] D.C. Lagoudas, "Shape Memory Alloys: Modeling and Engineering Applications", Springer Science + Business media, LLC, (2008).

[3] X. Chen, Z. Moumni, Y. He and W. Zhang, J. of the Mech. and Phys. of Solids, 64, (2014), 249-286.

[4] A. Sozinov, N. Lanska, A. Soroka, and W. Zou, Appl. phys. letters, 102 (2013), 021902.

[5] I. Karaman, B. Basaran, H.E. Karaca, A.I. Karsilayan and Y.I. Chumlyakov, Appl. phys. letters, 90, 17 (2007) p172505.

[6] L. Daniel, O. Hubert, N. Buiron, R. Billardon, J. of the Mech. and Phys. of Solids, 56 (2008), 1018-1042.

[7] A. Maynadier, D. Depriester, K. Lavernhe-Taillard, O. Hubert, Procedia Engineering, 10 (2011), 2208-2213.

[8] C. Jiang, Y. Muhammad, L. Deng, W. Wu and H. Xu, Acta Mat., 52 (2004), 2779-2785.

[9] L. Straka, O. Heczko, S.-P. Hannula, Scripta Mater, 548 (2006), 14971500

[10] O. Hubert and L. Daniel, J. of Magn. and Magn. Mater, 3207 (2008), 1412-1422.

[11] O. Heczko, J. Kopeek, D. Majtás and M. Landa, J. of Physics Conference Series, 303 (2011), 012081.

[12] O. Heczko, K. Jurek and K. Ullakko, J. of Magn. and Magn. Mater.,226230 (2001), 996-998. 